(2) Open Access Full Text Article

\title{
Icariside II ameliorates endothelial dysfunction by regulating the MAPK pathway via miR-I26/SPREDI in diabetic human cavernous endothelial cells
}

This article was published in the following Dove Press journal:

Drug Design, Development and Therapy

\author{
Hongen Lei' \\ Huixi $\mathrm{Li}^{2}$ \\ Long Tian' \\ Meng $\mathrm{Li}^{2}$ \\ Zhongcheng $\mathrm{Xin}^{2}$ \\ Xiaodong Zhang' \\ Ruili Guan² \\ 'Department of Urology, Beijing \\ Chao-Yang Hospital, Capital Medical \\ University, Beijing 100020, People's \\ Republic of China; ${ }^{2}$ Andrology \\ Center, Peking University First \\ Hospital, Peking University, Beijing \\ 100034, People's Republic of China
}

Correspondence: Ruili Guan

Andrology Center, Peking University First Hospital, Peking University, No 8 Xishiku Street, Xicheng District, Beijing 100034,

People's Republic of China

Tel +86 10 83228989

Fax +86I0 83222822

Email guanruili@bjmu.edu.cn

\section{Xiaodong Zhang}

Department of Urology, Beijing Chao-

Yang Hospital, Capital Medical University,

No 8 Gongren Tiyuchang Nanlu,

Chaoyang District, Beijing 100020,

People's Republic of China

Tel/fax +86 I0 $8523 \quad$ I247

Email zxdcyyy@I63.com
Aim: The aim of the study was to investigate whether miR-126, a regulator of MAPK signaling via targeting sprouty-related EVH1 domain-containing protein 1 (SPRED1) mRNA, is involved in the process by which icariside II (ICA II) ameliorates endothelial dysfunction in human cavernous endothelial cells (hCECs) exposed to a diabetic-like environment.

Materials and methods: Primary hCECs were isolated and divided into three groups, normal control, diabetes mellitus (DM), and DM treated with ICA II. The cell proliferation and migration abilities of the hCECs were examined. The expression levels of endothelial-related microRNAs and relative target mRNAs (SPRED1, phosphoinositol-3 kinase regulatory subunit 2 , and vascular cell adhesion molecule 1) of miR-126 were determined by real-time PCR. The protein expression of endothelial nitric oxide synthase, receptor for advanced glycation end products, and SPRED1, and MAPK signaling activities was determined by Western blot analysis. In addition, miR-126 agomir and antagomir were used for transfection into hCECs to further testify the association between miR-126 and its targeting mRNA SPRED1.

Results: hCECs induced with glucose plus advanced glycation end product-BSA showed a significant decrease in endothelial nitric oxide synthase, Ki-67, and miR-126 expression; a downregulated cell migration ability and an increased receptor for advanced glycation end products level. ICA II could partially reverse these changes. SPRED1 mRNA showed a contrary tendency with the miR-126-3p changes. The level of SPRED1 protein increased after the hCECs were induced with glucose plus advanced glycation end product-BSA, and ICA II could rescue its aberrant expression. In addition, the MAPK pathway was downregulated in the hCECs under diabetic conditions, and ICA II could partially enhance its signaling activities. miR-126 was obviously downregulated, and SPREDI was accordingly upregulated after miR-126 antagomir transfection, while ICA II treatment could recover the expressions of both miR-126 and SPRED1. Moreover, the upregulation of miR-126 and the inhibition of SPRED1 were noticed in the diabetic hCECs by further transfection with miR-126 agomir.

Conclusion: ICA II could ameliorate endothelial dysfunction by regulating the MAPK pathway via miR-126/SPRED1 in hCECs exposed to a diabetic-like environment, and ICA II might be a protective agent for endothelial function in diabetic ED.

Keywords: human cavernous endothelial cells, endothelial dysfunction, miR-126, diabetes mellitus, icariside II

\section{Introduction}

Erectile dysfunction (ED) is defined as the consistent inability to attain or maintain a penile erection of sufficient quality to perform satisfactory sexual intercourse, and diabetes mellitus (DM) is considered to be an important risk factor causing ED. ${ }^{1}$ For diabetic ED, the putative pathogenesis is always associated with multifactorial respects, 
such as hyperglycemia, accumulation of advanced glycation end products (AGEs), excess free fatty acids, and insulin resistance. ${ }^{2}$ Endothelial cells are essential components of the penile cavernous tissue in the penis, which could produce endothelial nitric oxide synthase (eNOS) to catalyze the production of nitric oxide, leading to the normal erection of the penis, ${ }^{3}$ while endothelial dysfunction plays a predominant role in the pathogenesis of diabetic ED. Icariside II (ICA II, $\mathrm{C}_{27} \mathrm{H}_{32} \mathrm{O}_{10}$, MW 514.54), the bioactive form of icariin, is isolated from Herba Epimedii, which has been demonstrated to have beneficial effects on diabetic ED in vivo and in vitro in our previous studies. ${ }^{4,5}$ Its therapeutic effects might be related with the morphological restoration of endothelial dysfunction, neuropathy, smooth muscle atrophy, and collagen deposition, but little is known about the underlying mechanisms, especially in the field of endothelial-related function.

MicroRNAs (miRNAs) are a class of noncoding RNAs (21-23 nucleotides in length) that regulate their target mRNA expression by specifically interacting with the $3^{\prime}$ untranslated regions of the mRNA, inhibiting protein translation or destabilizing the mRNA. MiRNAs are involved in many critical cell functions and play important roles in many diseases, such as cardiovascular diseases, kidney diseases, diabetes, and tumors. ${ }^{6}$ However, the question of whether endothelialrelated miRNAs are involved in the process by which ICA II regulates endothelial dysfunction needs further elucidation. miR-126 is highly enriched in endothelial cells, and it could mediate angiogenesis, homeostasis, and vascular integrity by mainly repressing its target genes sprouty-related EVH1 domain-containing protein 1 (SPRED1), phosphoinositol-3 kinase regulatory subunit $2(P I K 3 R 2)$, and vascular cell adhesion molecule 1 (VCAM1), all of which could regulate multiple signaling pathways to play important roles in maintaining endothelial cell function. ${ }^{7}$ At the present time, the role of miR-126 in the process by which ICA II regulates endothelial function in a diabetic ED model is worthy of investigation.

In this study, we aimed to investigate whether miR-126, a regulator of the MAPK signaling via targeting SPRED1 mRNA, is involved in the process by which ICA II ameliorates endothelial dysfunction in human cavernous endothelial cells (hCECs) exposed to $250 \mathrm{mg} / \mathrm{dL}$ glucose plus $200 \mu \mathrm{g} / \mathrm{mL}$ AGE-BSA, a diabetic-like environment, which could be used to mimic the cell microenvironment in diabetic patients.

\section{Materials and methods}

\section{hCECs isolation and culture}

Primary hCECs were isolated from human corpus cavernosum tissue in patients who received a penile prosthesis implantation by a modified method as previously described. ${ }^{5}$ Briefly, after rinsing with PBS, the tissue sample was cut into pieces and was digested in EGM-2 medium (cc-3156 and cc-4176, LONZA, Allendale, NJ, USA) supplemented with $1.5 \mathrm{U} / \mathrm{mL}$ dispase (17105-041, Invitrogen, Carlsbad, CA, USA) at $37^{\circ} \mathrm{C}$ for $6 \mathrm{~h}$. Then, after filtering through a $70 \mu \mathrm{m}$ cell strainer, the cells were collected by centrifugation at $1,000 \mathrm{rpm}$ for $5 \mathrm{~min}$ and were re-suspended and cultured in EGM-2 complete medium in a $\varphi 10 \mathrm{~cm}$ culture plate. When cultured to $80 \%$ confluence, the cells were trypsinized and incubated with an anti-CD31 antibody (1:200, Abcam, Cambridge, MA, USA) at $4^{\circ} \mathrm{C}$ for $45 \mathrm{~min}$. After washing twice with the autoMACS Pro Washing Solution (130-092987, MACS, Bergisch Gladbach, Germany), the cells were incubated with anti-mouse IgG microbeads (130-048-402, MACS) at $4{ }^{\circ} \mathrm{C}$ for $15 \mathrm{~min}$ and were washed twice with the autoMACS Pro Washing Solution again. Finally, the CD31positive cells were selected using a MACS selection system (130-042-401 and 130-042-302, MACS) according to the manufacturer's protocol. hCECs of passages 2-6 were used in the study. To conduct all the procedures, we obtained a written informed consent from the patients, and the study was approved by the Medical Ethics Committee of Beijing Chao-Yang Hospital, Capital Medical University.

Purified hCECs were cultured in EGM-2 medium (cc-3156 and cc-4176; LONZA) supplemented with 20\% fetal bovine serum (Gibco, Grand Island, NY, USA) and $2 \mathrm{mM}$ of L-glutamine. All the cells were cultured at $37^{\circ} \mathrm{C}$ with 5\% $\mathrm{CO}_{2}$ in a humidified incubator (Forma 3110; Thermo Scientific, Lincoln, NE, USA).

\section{Diabetes induction and ICA II treatment}

The AGE-BSA used in this study was purchased from BioVision, Inc. (2221-BSA, Milpitas, CA, USA), and hCECs, that were $50 \%-60 \%$ confluent, were used for the experiments. hCECs were induced in medium containing $200 \mu \mathrm{g} / \mathrm{mL}$ BSA (AMRESCO, Solon, OH, USA) for the normal control (NC) group or in a diabetic-like environment medium containing $250 \mathrm{mg} / \mathrm{dL}$ glucose (Gibco-Invitrogen, Grand Island, NY, USA) plus $200 \mu \mathrm{g} / \mathrm{mL}$ AGE-BSA for the DM group for $96 \mathrm{~h},{ }^{8}$ and ICA II (purity: 98\%, 113558-15-9; Herbest, Xi'an, People's Republic of China) at $0.1,1$, and $10 \mu \mathrm{M}$ was added to the DM group $1 \mathrm{~h}$ after the induction with high glucose plus AGE-BSA to stimulate the cells for an additional $95 \mathrm{~h}$ for the DM treated with ICA II (DM+ICA II) group.

\section{Western blot analysis}

The hCECs were lysed in RIPA lysis buffer (CW2333, CWBIO, Beijing, People's Republic of China) containing a 
protease and phosphatase inhibitor cocktail (Sigma-Aldrich, St. Louis, MO, USA). The soluble lysate was mixed with $5 \times$ loading buffer (CW0053, CWBIO) and boiled for $10 \mathrm{~min}$. The cell lysates containing $20 \mu \mathrm{g}$ of protein were electrophoresed by sodium dodecyl sulfate polyacrylamide gel electrophoresis and were then transferred to a polyvinylidene fluoride membrane (Millipore Corp, Bedford, MA, USA). After being blocked with 5\% skimmed milk for $1 \mathrm{~h}$ at room temperature and incubating overnight at $4^{\circ} \mathrm{C}$ with primary antibodies against eNOS (1:500, BD Transduction Laboratories, San Jose, CA, USA), receptor for advanced glycation end products (RAGE; 1:500, Abcam), SPRED1 (1:500, Abcam), c-Raf (1:1,000, CST, Danvers, MA, USA), phospho-MEK1/2 (1:1,000, CST), MEK1/2 (1:1,000, CST), phospho-Erk1/2 (1:1,000, CST), Erk1/2 (1:1,000, CST), and GAPDH (1:10,000, CWBIO). The membrane was immersed in an HRP-conjugated secondary antibody, followed by chemiluminescence detection using a chemiluminescencecompatible digital imaging system (C-DiGit Blot Scanner, LI-COR Biosciences, Cambridge, UK). The integrated density value of each protein band from the Western blot images was analyzed with ImageJ software (version 1.46r, ImageJ, NIH, Bethesda, MD, USA).

\section{Immunofluorescence staining}

The immunofluorescence staining was carried out as following: hCECs were fixed in $4 \%$ cold paraformaldehyde at $4{ }^{\circ} \mathrm{C}$ for $15 \mathrm{~min}$ and were immersed in Triton X-100 for $5 \mathrm{~min}$ and in $5 \%$ goat serum for $1 \mathrm{~h}$. Then, a primary antibody against Ki-67 (1:400, Abcam) was used for overnight incubation at $4{ }^{\circ} \mathrm{C}$. After the cells were washed three times with PBS, they were then incubated with a fluorescein-conjugated secondary antibody at room temperature for $2 \mathrm{~h}$. Finally, after 4',6-diamidino-2-phenylindole staining for $10 \mathrm{~min}$, the cell images were captured using a digital camera (Leica DM2500, Leica Microsystems, Wetzlar, Germany).

\section{Scratch wound assay}

When the cells reached a confluence of $30 \%-40 \%$ in six-well plates, the normal hCECs were wounded using a $200 \mu \mathrm{L}$ yellow micropipette tip. Then, the cells were washed three times with PBS and were induced in EGM-2 medium without fetal bovine serum for 4 days, and the hCECs from the NC group, DM group, and DM+ICA II group were induced in the corresponding media as previously described, and ICA II, at a final concentration of $10 \mu \mathrm{M}$, was used in this assay. Finally, after the cells were fixed in $4 \%$ paraformaldehyde at $4^{\circ} \mathrm{C}$ for $15 \mathrm{~min}$ and stained with a $1 \%$ crystal violet solution at room temperature for $30 \mathrm{~min}$, the cell images were captured using a digital camera (Leica DM2500, Leica Microsystems).

\section{RNA isolation and real-time PCR}

Total RNA (including miRNA) was extracted from the cells using a miRcute miRNA Isolation Kit (DP501, Tiangen Biotech, Beijing, People's Republic of China). The cDNA, for miRNA detection, was synthesized from $2 \mu \mathrm{g}$ of total RNA using a miRcute miRNA First-Strand cDNA Synthesis Kit (KR201, Tiangen Biotech), and the cDNA, for target gene detection, was synthesized from $2 \mu \mathrm{g}$ of total RNA using a SuperQuickRT cDNA Kit (CW2381, CWBIO). The relative miRNA expression was quantified by a SYBR Green miRcute miRNA qPCR Detection Kit (FP401, Tiangen Biotech) and was normalized according to hsa-U6 expression, and the relative target mRNA expression was quantified by a TransStart SYBR Green qPCR SuperMix (AQ131, TransGen Biotech, Beijing, People's Republic of China) and was normalized according to the GAPDH expression. The forward primers for the miRNAs (Table 1), a universal reverse primer, and hsa-U6 primers were purchased from TransGen Biotech. The primers for the target gene mRNAs (Table 2) were synthesized by Sangon Biotech, Shanghai, People's Republic of China. The PCR conditions for both detections were $95^{\circ} \mathrm{C}$ for $20 \mathrm{~s}$ and $60^{\circ} \mathrm{C}$ for $34 \mathrm{~s}$. The relative levels of the miRNAs and the target mRNAs were defined from the threshold cycle (Ct) values calculated by the $2 \mathrm{~s} \Delta \Delta \mathrm{Ct}$ method.

\section{Transfecting RNA oligonucleotides into hCECs}

To overexpress or suppress miR-126-3p in hCECs, RNA oligonucleotides for miR-126 agomir and antagomir were synthesized by GenePharm, Shanghai, People's Republic of China, and a scrambled oligonucleotide served as the control. When the cells reached a confluence of 30\%-40\% in six-well plates, the normal hCECs were transfected with a final concentration of $5 \mathrm{nM}$ of RNA oligonucleotides using a transfection agent purchased from GenePharm.

Table I The forward primers of miRNAs

\begin{tabular}{ll}
\hline miRNAs & Forward primer $\left(5^{\prime}-3^{\prime}\right)$ \\
\hline hsa-miR-126-3p & TCGTACCGTGAGTAATAATGCG \\
hsa-miR-126-5p & CATTATTACTTTTGGTACGCG \\
hsa-miR-15a-5p & TAGCAGCACATAATGGTTTGTG \\
hsa-miR-15a-3p & CAGGCCATATTGTGCTGCCTCA \\
hsa-miR-16-5p & TAGCAGCACGTAAATATTGGCG \\
hsa-miR-145-5p & GTCCAGTTTTCCCAGGAATCCCT \\
hsa-miR-155-5p & TTAATGCTAATCGTGATAGGGGT \\
hsa-miR-200b-3p & TAATACTGCCTGGTAATGATGA \\
\hline
\end{tabular}

Abbreviation: miRNA, microRNA. 
Table 2 The primers of target gene mRNAs

\begin{tabular}{|c|c|c|}
\hline mRNAs & Forward primer $\left(5^{\prime}-3^{\prime}\right)$ & Reversed primer $\left(5^{\prime}-3^{\prime}\right)$ \\
\hline SPRED I & GAGACAGTTGTTACCAGTGAGCC & CCATACTTCTGCTCTGAATGTCC \\
\hline PIK3R2 & TTAAGGTCTATCACCAGCAGTACCA & ATCTCTTTCTCGTTGCCCTCAC \\
\hline VCAMI & GCGGGAGTATATGAATGTGAATCTAA & GCTACACTTTTGACTTCTGTGCTTCT \\
\hline GAPDH & ACGGATTTGGTCGTATTGGG & TGATTTTGGAGGGATCTCGC \\
\hline
\end{tabular}

Abbreviations: SPREDI, sprouty-related EVHI domain-containing protein I; PIK3R2, phosphoinositol-3 kinase regulatory subunit 2; VCAMI, vascular cell adhesion molecule I.

After $6 \mathrm{~h}$, the cells were washed three times with PBS and were induced in total EGM-2 medium for another 4 days. The hCECs from the NC group, DM group, and DM+ICA II group were induced in the corresponding media as previously described, and ICA II, at a final concentration of 10 $\mu \mathrm{M}$, was used in this assay.

\section{Statistical analysis}

The data are shown as mean \pm SD. Comparisons between the treatment groups and control groups were made based on Student's unpaired $t$-test. Multiple comparisons were determined using a one-way ANOVA followed by the StudentNewman-Keuls test. All the calculations were performed using SPSS statistical software (version 13.0, SPSS, Chicago, IL, USA). $P$-values $<0.05$ were considered significant.

\section{Results}

\section{Diabetic hCECs induction and the effects of ICA II on cell function in diabetic hCECs}

After the hCECs were induced in a diabetic-like environment, with media containing $250 \mathrm{mg} / \mathrm{dL}$ glucose plus $200 \mu \mathrm{g} / \mathrm{mL}$ AGE-BSA (DM) for $96 \mathrm{~h}$, Western blot analysis showed that eNOS significantly decreased and RAGE increased, and this demonstrated that diabetic hCECs were successfully induced. Then, different concentrations $(0.1,1$, and $10 \mu \mathrm{M})$ of ICA II were added to investigate the effects of ICA II on the diabetic hCECs, and ICA II rescued the protein expression of eNOS and RAGE in the diabetic hCECs to a certain extent (Figure 1A and B). ICA II, at a concentration of $10 \mu \mathrm{M}$, which exerted the optimal beneficial effect for endothelial dysfunction based on the eNOS and RAGE changes, was used in the subsequent studies, unless indicated otherwise. Meanwhile, the results from the immunofluorescence staining and scratch wound assay showed that Ki-67 expression and the cell migration ability were downregulated after the hCECs were induced with glucose plus AGE-BSA, and ICA II partially reversed the morphological changes of cell proliferation and migration in the diabetic hCECs (Figure 1C and D).

\section{Endothelial-related miRNA screening and the effects of ICA II on miR-I26/SPRED I expression in diabetic hCECs}

MiRNAs regulate target genes to modulate signaling pathways, and we first screened eight endothelial-related miRNAs (miR-126-3p, miR-126-5p, miR-15a-5p, miR-15a-3p, miR-16-5p, miR-145-5p, miR-155-5p, and miR-200b-3p) that may be involved in the development of diabetic ED according to the literature. ${ }^{7,9-11}$ The real-time PCR results showed that miR-126-3p and miR-155-5p were differentially expressed among the NC, DM, and DM+ICA II groups (Figure 2A). The role of miR-155-5p and its target gene eNOS has been investigated thoroughly in endothelial dysfunction cells. ${ }^{12}$ Next, we aimed to study miR-126-3p and its target genes in light of its putative role in vascular integrity and angiogenesis in endothelial cells. ${ }^{713}$ The expression profiles of SPRED1, PIK3R2, and VCAM1, the main target genes of miR-126-3p, were further detected, and the results showed that SPRED1 had a contrary tendency with the miR-126-3p changes (Figure 2B), which demonstrated that miR-126/SPRED1 may play an important role in the process of by which ICA II reverses the morphological changes in diabetic hCECs.

\section{Effects of ICA II on regulating the MAPK pathway activities in diabetic hCECs}

The activation of MAPK signaling stimulates the transcription of genes involved in angiogenesis, and a gain of miR-126 function enhances the angiogenic signaling of MAPK by repressing the expression of SPRED $1 .{ }^{13}$ Thus, the protein expression of SPRED1 and key molecules in the MAPK pathway (c-Raf, p-MEK1/2, MEK1/2, p-Erk1/2, and Erk1/2) was tested in this model. The results showed that the level of SPRED1 protein increased after the hCECs were induced with glucose plus AGE-BSA, and ICA II rescued its aberrant expression (Figure 3). In addition, the MAPK pathway was downregulated in the hCECs under diabetic conditions, and ICA II partially enhanced its signaling activities (Figure 3). These results suggested that the MAPK pathway may be 
A

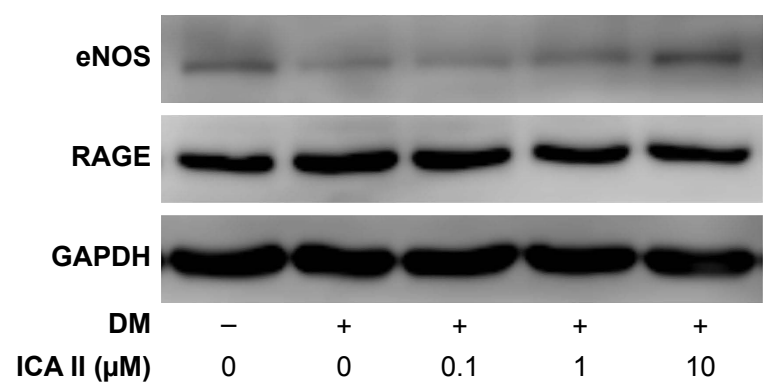

B

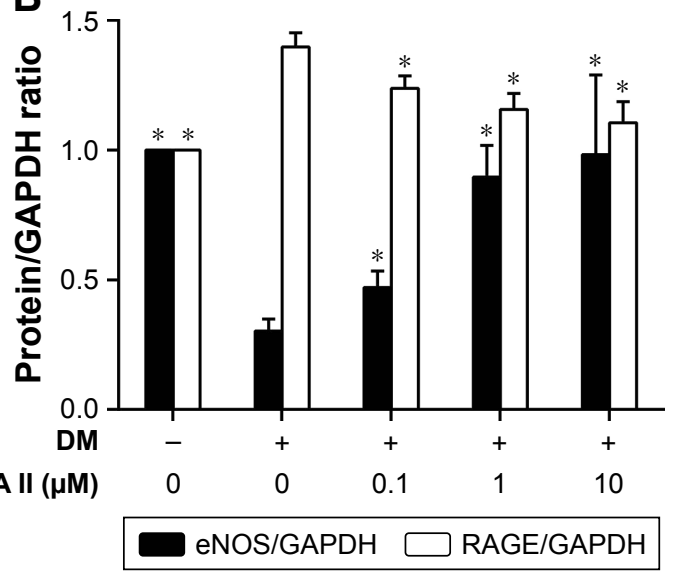

C
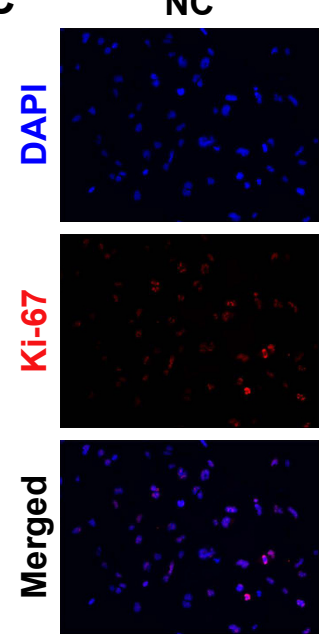

DM
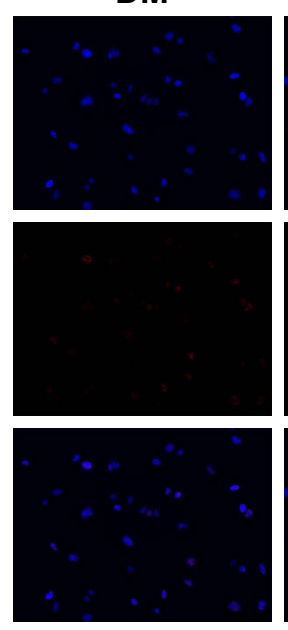

DM+ICA II
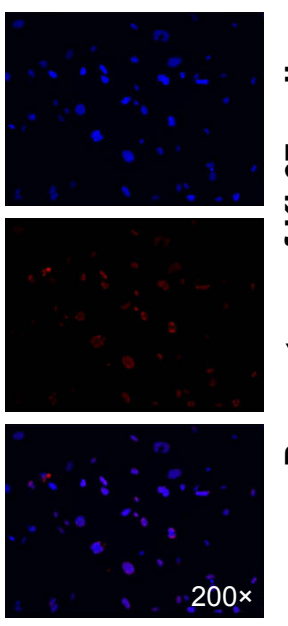
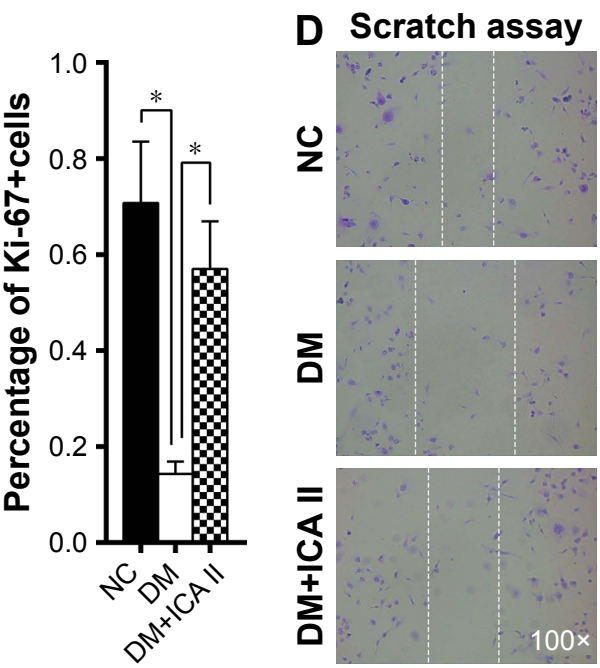

Figure I Diabetic hCEC identification and the effect of ICA II on cell proliferation and migration in diabetic hCECs.

Notes: (A) The protein expression of eNOS and RAGE was assessed by Western blot. (B) The quantitative data of the eNOS and RAGE protein expression were normalized to GAPDH levels compared with the NC group as I. (C) Ki-67 expression for cell proliferation ability was determined by immunofluorescence, and the cell nuclei were labeled with DAPI. (D) The cell proliferation ability was determined by a scratch migration assay, and the cell migration spans are shown. The data are presented as the mean \pm SD of three independent experiments. $* P<0.05$ when compared with the DM group.

Abbreviations: hCEC, human cavernous endothelial cell; ICA II, icariside II; eNOS, endothelial nitric oxide synthase; RAGE, receptor for advanced glycation end products; NC, normal control; DAPI, 4',6-diamidino-2-phenylindole; DM, diabetes mellitus; DM+ICA II, DM treated with ICA II.

involved in the regulation of endothelial function by ICA II in hCECs.

\section{Further evaluation of the influence of miR-I26-3p on SPRED I expression in hCECs}

Next, normal hCECs were transfected with miR-126 antagomir to inhibit miR-126 expression, and then ICA II was further added to investigate its effect. The results showed that miR-126 was obviously downregulated, and SPRED1 was accordingly upregulated after miR-126 antagomir transfection, while ICA II treatment could recover the expression of both Mir126 and SPRED1 (Figure 4A). Moreover, the upregulation of miR-126 expression and the inhibition of SPREDI expression were noted in the diabetic hCECs by further transfecting with miR-126 agomir (Figure 4B), suggesting that miR-126/ SPRED1 signaling was involved in the process by which ICA II regulates endothelial function in diabetic hCECs.

\section{The possible mechanism by which} ICA II regulates endothelial cell function involves regulating the MAPK pathway via miR-I 26/SPREDI in diabetic hCECs

It is reported that miR-126 plays a key role in vascular integrity and angiogenesis; miR-126 could repress its target protein expression of SPRED1, which is a negative regulator of Ras/MAPK signaling. ${ }^{13}$ In this study, we found that 
A

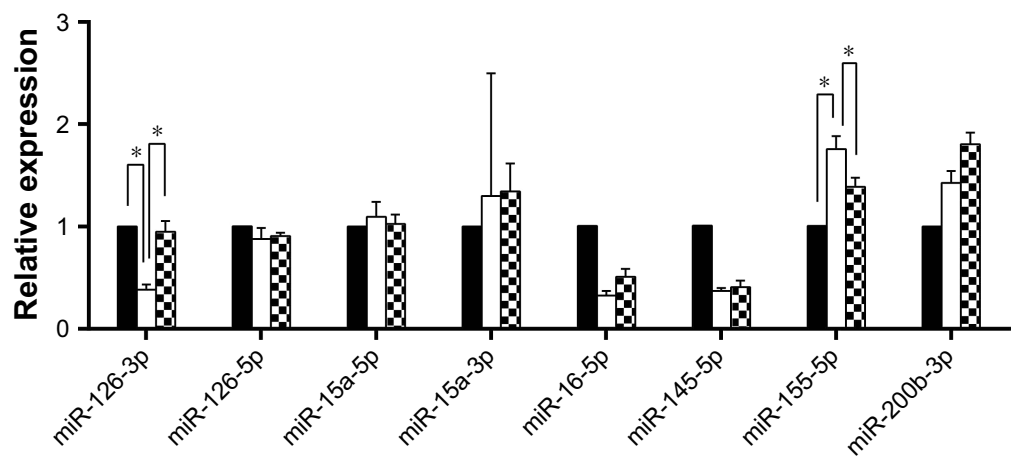

B

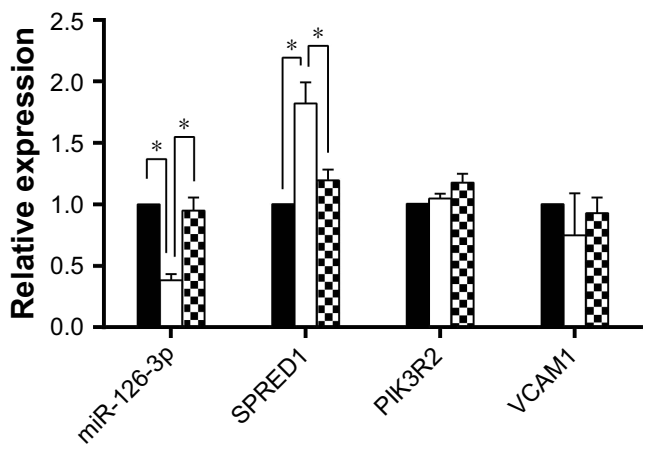

Figure 2 Effect of ICA II on endothelial miRNAs and miR-I26/SPREDI expression in diabetic hCECs.

Notes: (A) Endothelial miRNA candidates (miR-I26-3p, miR-126-5p, miR-I5a-5p, miR-I5a-3p, miR-I6-5p, miR-I45-5p, miR-I55-5p, and miR-200b-3p) were assessed in a diabetic hCEC model with or without ICA II treatment by real-time PCR, and the quantitative data of the miRNAs were normalized to hsa-U6 levels. (B) The effect of ICA II on miR-I26 and the expression of its target gene mRNAs (SPREDI, PIK3R2, and VCAMI) were assessed by real-time PCR, and the quantitative data of the target genes were normalized to GAPDH levels. The data are presented as the mean \pm SD of three independent experiments. $* P<0.05$ when compared with the $D M$ group.

Abbreviations: ICA II, icariside II; miRNA, microRNA; SPREDI, sprouty-related EVHI domain-containing protein I; hCEC, human cavernous endothelial cells; PIK3R2, phosphoinositol-3 kinase regulatory subunit 2; VCAMI, vascular cell adhesion molecule I; NC, normal control; DM, diabetes mellitus; DM+ICA II, DM treated with ICA II.

ICA II mediated the miR-126/SPRED1 regulation by transfection with miR-126 antagomir, and the overexpression of miR-126 could inhibit SPRED1 expression in diabetic hCECs by transfection with miR-126 agomir. Furthermore,
ICA II treatment could rescue the activities of the MAPK signaling pathway, which were inhibited in a diabetic condition. In summary, we believe that ICA II may promote cell proliferation and migration by upregulating the MAPK
A

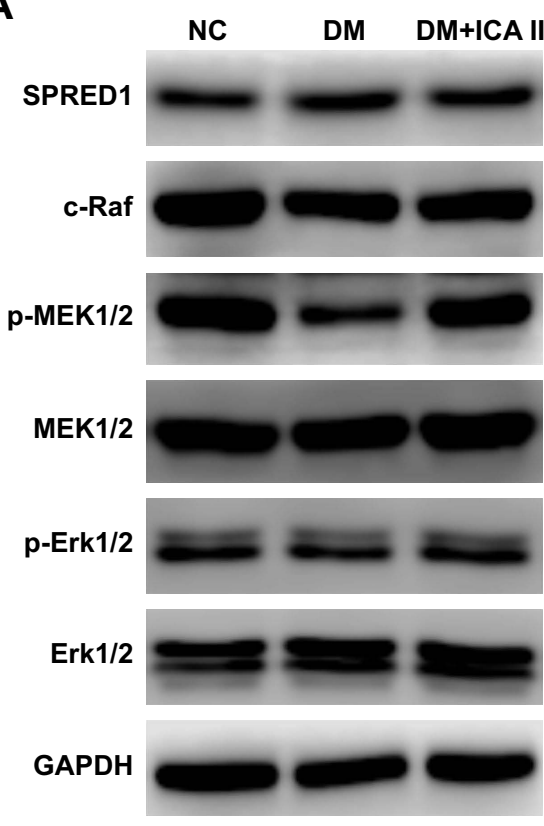

B

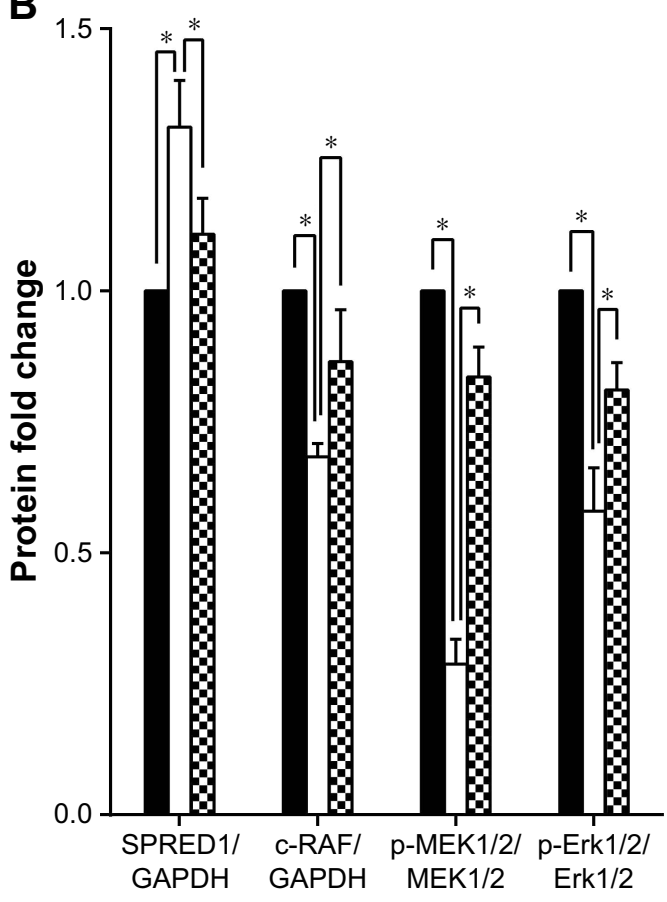

$\square \mathrm{NC} \square \mathrm{DM} \square \mathrm{DM}+\mathrm{ICA} I \mathrm{I}$

Figure 3 Effects of ICA II on regulating protein expression levels in the MAPK pathway in diabetic hCECs.

Notes: (A) The protein expression of SPREDI, c-Raf, phosphorylated MEK I/2, MEK I/2, Erk I/2, and phosphorylated ErkI/2 in the MAPK pathway were assessed by Western blot. (B) The quantitative data of the protein expression levels in the MAPK pathway were compared with NC group as I. The data are presented as the mean \pm SD of three independent experiments. ${ }^{*} P<0.05$ when compared with the DM group.

Abbreviations: ICA II, icariside II; hCECs, human cavernous endothelial cells; SPRED I, sprouty-related EVHI domain-containing protein I; NC, normal control; DM, diabetes mellitus; DM+ICA II, DM treated with ICA II. 

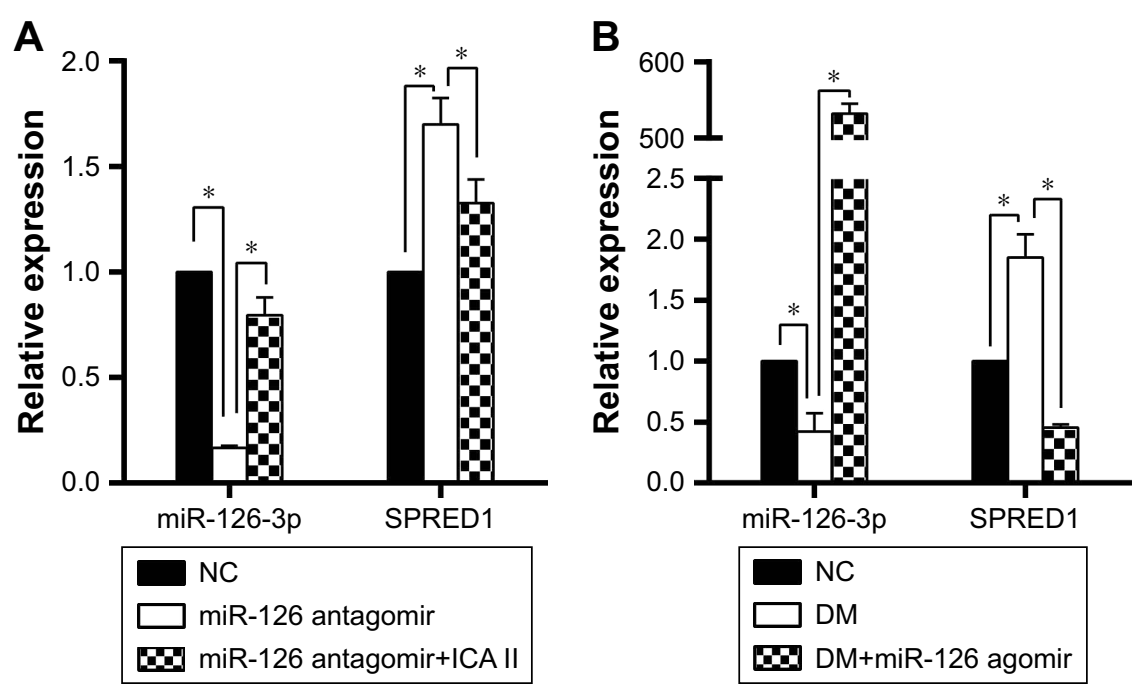

C<smiles>CC(C)=CCc1oc(-c2ccc(O)cc2)c(OC2C3CCC(C3)C2C)c(=O)c1O</smiles>
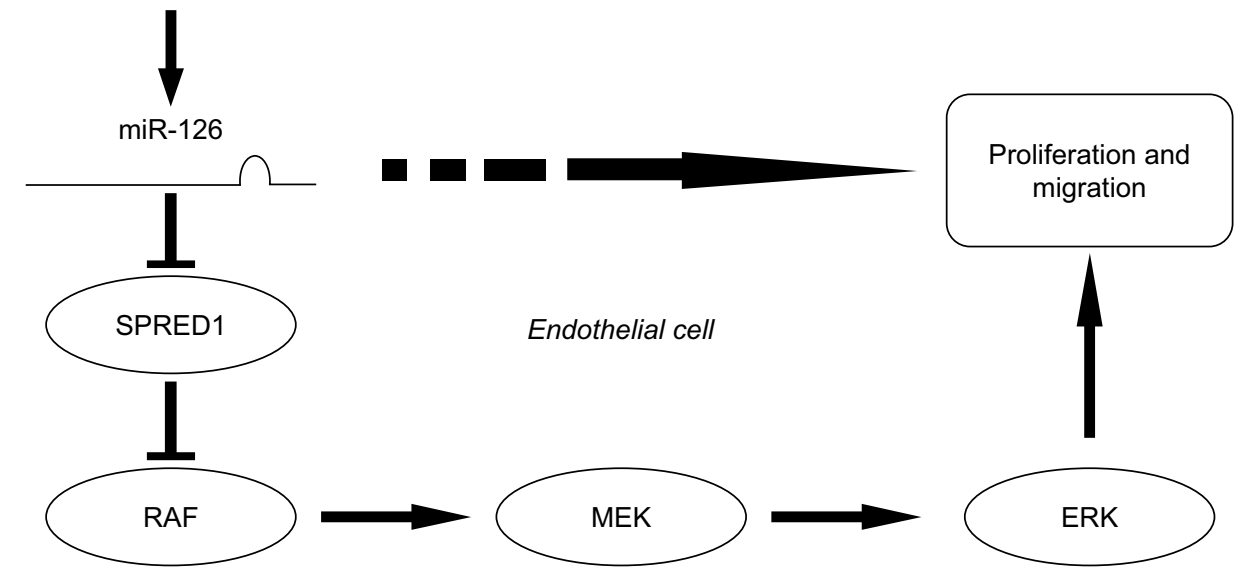

Figure 4 The influence of miR-I26-3p on SPREDI regulation and the hypothetical scheme of ICA II on endothelial cell function in diabetic hCECs.

Notes: (A and B) miR-I26 and SPREDI mRNA expression was assessed by real-time PCR. The quantitative data for miR-I26 were normalized to the hsa-U6 level, and the quantitative data for SPREDI mRNA were normalized to the GAPDH level. The data are presented as the mean \pm SD of three independent experiments. $* P<0.05$ when compared with the miR-I26 antagomir group or the DM group. (C) It is putative that miR-I26 represses its target gene expression, SPRED I, which is a negative regulator of MAPK signaling. We suppose that ICA II may promote cell proliferation and migration by upregulating the MAPK signaling pathway via miR-I26/SPREDI in diabetic hCECs. Abbreviations: SPREDI, sprouty-related EVHI domain-containing protein I; ICA II, icariside II; DM, diabetes mellitus; hCECs, human cavernous endothelial cells; NC, normal control.

signaling pathway via miR-126/SPRED1 in diabetic hCECs (Figure 4C).

\section{Discussion}

Type I and type II diabetes cause many complications, including heart attack, stroke, kidney failure, blindness, and ED. Penile endothelial dysfunction is one of the most important pathological factors in diabetic ED. ${ }^{14,15}$ Persistent hyperglycemia in diabetes leads to the formation of AGEs, which can bind to the RAGE to generate cell stress, cellular dysfunction, and target organ damage, leading to the occurrence of DM complications. ${ }^{16}$ In patients with diabetes, hyperglycemia and AGEs coexist to damage endothelial cell function, which is considered as the most significant cause for diabetic impotency. ${ }^{17}$

An endothelial dysfunction cell model has been established by the induction of high glucose or AGEs, separately, in vitro to study the endothelial function. ${ }^{18,19}$ In a previous study, stimulation with normal glucose $(5 \mathrm{mM})$, normal glucose plus mannitol (20 mM), and high glucose (20 mM) 
was used to study the interference effect of osmotic pressure in the endothelial dysfunction model establishment of hCECs. ${ }^{5}$ The results showed no significant differences between normal glucose $(5 \mathrm{mM})$ and normal glucose plus mannitol (20 mM) groups, while significant differences were found in the hCECs cultured in the high glucose $(20 \mathrm{mM})$ and normal glucose $(5 \mathrm{mM})$ groups, demonstrating that the influence of osmolarity can be neglected during the diabetic model construction induced by high glucose under a concentration of $\leq 20 \mathrm{mM} .{ }^{5}$ High glucose plus AGEs could induce significantly differential expression of metabolic genes in endothelial cells, including endothelial inflammatory-related genes in the NFKB pathway, thioredoxin interacting protein, and many miRNAs. ${ }^{20,21}$ The commonly used stimulation concentration is $250 \mathrm{mg} / \mathrm{dL}$ glucose plus $200 \mu \mathrm{g} / \mathrm{mL}$ AGEBSA, which is similar to that found in the blood of diabetic patients. ${ }^{8}$ In the present study, we adopted the condition of $250 \mathrm{mg} / \mathrm{dL}(\sim 18.88 \mathrm{mM})$ glucose plus $200 \mu \mathrm{g} / \mathrm{mL}$ AGE-BSA stimulation to construct an endothelial dysfunction model of hCECs. Considering that there is no impact of high glucose on the osmolarity, we did not use the normal glucose plus mannitol group in the current study.

The establishment of the in vitro endothelial dysfunction model was confirmed by the eNOS decrease, RAGE upregulation, and cell proliferation inhibition in the hCECs exposed to high glucose plus AGEs, and these changes were in accordance with experimental results reported in the literature. $^{22}$ ICA II, as a potential Chinese herbal monomer extract, rescues the penile cavernous endothelial dysfunction and effectively improves erectile function, ${ }^{23}$ but little is known about its underlying molecular mechanisms. No work has been reported about the relationship between miRNAs and ICA II interference in diabetic hCECs. miRNAs are decisive for endothelial cell gene expression and function in various pathological conditions associated with cardiovascular diseases. ${ }^{24}$ Recently, a study showed that 31 mature human miRNAs in HUVECs were significantly changed under a combined stimulation of high glucose and AGEs mimicking a diabetic-like environment, of which 18 were upregulated and 13 were downregulated, revealing that multiple miRNAs may be involved in the process of endothelial dysfunction. ${ }^{8}$ In this study, we first screened some endothelial-related miRNAs (miR-126-3p, miR-126-5p, miR-15a-5p, miR-15a-3p, miR16-5p, miR-145-5p, miR-155-5p, and miR-200b-3p), which may be involved in the development of diabetic ED. ${ }^{7,9-11}$ miR-126 plays a putative role in vascular integrity and angiogenesis in endothelial cells $;^{7} \mathrm{miR}-15 \mathrm{a}$ and $\mathrm{miR}-16$ affect angiogenesis by targeting $V E G F ;{ }^{9} \mathrm{miR}-126, \mathrm{miR}-145$, and miR-155, acting in atherosclerosis, may provide novel treatment options for cardiovascular diseases, ${ }^{10}$ and miR-200b is suppressed in AGE-induced endothelial cell injury. ${ }^{11}$ Our results showed that miR-126-3p and miR-155-5p were differentially expressed among the groups. The role of miR-155-5p and its target gene eNOS has been investigated thoroughly in endothelial dysfunction cells, and eNOS is a direct target of miR-155-5p and plays an important role in maintaining cardiovascular homeostasis. ${ }^{12}$ Thus, we focused on studying miR-126, which is aberrantly expressed in endothelial cells and plays a crucial role in maintaining the normal function of blood vessels, especially in mediating endothelial angiogenesis. ${ }^{25}$ Moreover, our results showed that ICA II treatment could improve the abovementioned endothelial indexes to a large extent. The overexpression of miR-126, by transfecting with miR-126 agomir, in diabetic hCECs could reduce the expression of SPRED1. The relationship between miR-126 and SPRED1 was further confirmed by treating the cells with miR-126 antagomir, while ICA II could reverse the effects by upregulating miR-126 and downregulating SPRED1. These results demonstrated that ICA II may be involved in the process of endothelial function restoration in diabetic ED via miR-126 and its target gene SPRED1, although transfection profiles of different concentrations of miR-126 antagomir and agomir were not performed. It is reported that miR-126 represses the expression of its target gene SPRED1, and SPRED1 protein is an intracellular inhibitor of the Ras/MAPK signaling pathway. ${ }^{26}$ By Western blot analysis, we confirmed that upregulated SPRED1 could negatively manipulate the MAPK pathway, and ICA II could activate the MAPK pathway via miR-126/SPRED1 signaling. To sum up, ICA II could promote cell proliferation and migration by upregulating the MAPK signaling pathway via miR-126/SPRED1 in diabetic hCECs, which may reveal the potential protective role of ICA II for endothelial dysfunction. ICA II might be a protective agent for endothelial function in diabetic ED in the future.

However, the molecular mechanisms of the reaction between ICA II and miR-126 and the effects of ICA II on the MAPK pathway are not fully elucidated. The specific role of miR-126/SPRED1 and MAPK signaling on the morphology and expression of various proteins in the diabetic ED model and how ICA II could improve endothelial function in diabetic hCECs still need further investigation.

\section{Acknowledgment}

This work was supported by the National Natural Science Foundation of China (No 81401194) and funded by the China Postdoctoral Science Foundation (No 2017M620832). 


\section{Disclosure}

The authors report no conflicts of interest in this work.

\section{References}

1. Shamloul R, Ghanem H. Erectile dysfunction. Lancet. 2013;381(9861): 153-165.

2. Kamenov ZA. A comprehensive review of erectile dysfunction in men with diabetes. Exp Clin Endocrinol Diabetes. 2015;123(3):141-158.

3. Lue TF. Erectile dysfunction. $N$ Engl J Med. 2000;342(24):1802-1813.

4. Zhou F, Xin H, Liu T, et al. Effects of icariside II on improving erectile function in rats with streptozotocin-induced diabetes. $J$ Androl. 2012;33(5):832-844.

5. Li H, Xu Y, Guan R, et al. Icariside II prevents high-glucose-induced injury on human cavernous endothelial cells through Akt-eNOS signaling pathway. Andrology. 2015;3(2):408-416.

6. Mendell JT, Olson EN. MicroRNAs in stress signaling and human disease. Cell. 2012;148(6):1172-1187.

7. Fish JE, Santoro MM, Morton SU, et al. miR-126 regulates angiogenic signaling and vascular integrity. Dev Cell. 2008;15(2):272-284.

8. Zitman-Gal T, Green J, Pasmanik-Chor M, Golan E, Bernheim J, Benchetrit S. Vitamin D manipulates miR-181c, miR-20b and miR-15a in human umbilical vein endothelial cells exposed to a diabetic-like environment. Cardiovasc Diabetol. 2014;13:8.

9. Sun CY, She XM, Qin Y, et al. miR-15a and miR-16 affect the angiogenesis of multiple myeloma by targeting VEGF. Carcinogenesis. 2013; 34(2):426-435.

10. Wei Y, Nazari-Jahantigh M, Neth P, WeberC, Schober A. MicroRNA-126, -145, and -155: a therapeutic triad in atherosclerosis? Arterioscler Thromb Vasc Biol. 2013;33(3):449-454.

11. Wu XD, Liu WL, Zeng K, et al. Advanced glycation end products activate the miRNA/RhoA/ROCK2 pathway in endothelial cells. Microcirculation. 2014;21(2):178-186.

12. Sun HX, Zeng DY, Li RT, et al. Essential role of microRNA-155 in regulating endothelium-dependent vasorelaxation by targeting endothelial nitric oxide synthase. Hypertension. 2012;60(6):1407-1414.

13. Wang S, Aurora AB, Johnson BA, et al. The endothelial-specific microRNA miR-126 governs vascular integrity and angiogenesis. Dev Cell. 2008;15(2):261-271.

14. Castela Â, Costa C. Molecular mechanisms associated with diabetic endothelial-erectile dysfunction. Nat Rev Urol. 2016;13(5):266-274.

15. Thorve VS, Kshirsagar AD, Vyawahare NS, Joshi VS, Ingale KG, Mohite RJ. Diabetes-induced erectile dysfunction: epidemiology, pathophysiology and management. J Diabetes Complications. 2011; 25(2):129-136.
16. Ramasamy R, Yan SF, Schmidt AM. Receptor for AGE (RAGE): signaling mechanisms in the pathogenesis of diabetes and its complications. Ann N Y Acad Sci. 2011;1243:88-102.

17. Neves D. Advanced glycation end-products: a common pathway in diabetes and age-related erectile dysfunction. Free Radic Res. 2013; 47 Suppl 1:49-69.

18. Li Y, Zhou Q, Pei C, et al. Hyperglycemia and advanced glycation end products regulate miR-126 expression in endothelial progenitor cells. J Vasc Res. 2016;53(1-2):94-104.

19. Tang ST, Wang F, Shao M, Wang Y, Zhu HQ. MicroRNA-126 suppresses inflammation in endothelial cells under hyperglycemic condition by targeting HMGB1. Vascul Pharmacol. 2017;88:48-55.

20. Schulze PC, Yoshioka J, Takahashi T, He Z, King GL, Lee RT. Hyperglycemia promotes oxidative stress through inhibition of thioredoxin function by thioredoxin-interacting protein. J Biol Chem. 2004;279(29): 30369-30374

21. Zitman-Gal T, Golan E, Green J, Bernheim J, Benchetrit S. Vitamin D receptor activation in a diabetic-like environment: potential role in the activity of the endothelial pro-inflammatory and thioredoxin pathways. J Steroid Biochem Mol Biol. 2012;132(1-2):1-7.

22. Ren X, Ren L, Wei Q, Shao H, Chen L, Liu N. Advanced glycation end-products decreases expression of endothelial nitric oxide synthase through oxidative stress in human coronary artery endothelial cells. Cardiovasc Diabetol. 2017;16(1):52.

23. Zhang J, Li AM, Liu BX, et al. Effect of icariside II on diabetic rats with erectile dysfunction and its potential mechanism via assessment of AGEs, autophagy, mTOR and the NO-cGMP pathway. Asian J Androl. 2013;15(1):143-148.

24. Nemecz M, Alexandru N, Tanko G, Georgescu A. Role of microRNA in endothelial dysfunction and hypertension. Curr Hypertens Rep. 2016;18(12):87.

25. Jansen F, Yang X, Hoelscher M, et al. Endothelial microparticlemediated transfer of microRNA-126 promotes vascular endothelial cell repair via SPRED1 and is abrogated in glucose-damaged endothelial microparticles. Circulation. 2013;128(18):2026-2038.

26. Pasmant E, Gilbert-Dussardier B, Petit A, et al. SPRED1, a RAS MAPK pathway inhibitor that causes Legius syndrome, is a tumour suppressor downregulated in paediatric acute myeloblastic leukaemia. Oncogene. 2015;34(5):631-638.

\section{Publish your work in this journal}

Drug Design, Development and Therapy is an international, peerreviewed open-access journal that spans the spectrum of drug design and development through to clinical applications. Clinical outcomes, patient safety, and programs for the development and effective, safe, and sustained use of medicines are the features of the journal, which

\section{Dovepress}

has also been accepted for indexing on PubMed Central. The manuscript management system is completely online and includes a very quick and fair peer-review system, which is all easy to use. Visit http://www.dovepress.com/testimonials.php to read real quotes from published authors. 